\title{
Study of periodic processes of changing the number of innovations in relation to Kondratiev cycles
}

\author{
Sofia Diakonova ${ }^{1, *}$, Stepan Artyshchenko ${ }^{1}$, Daria Sysoeva $^{1}$, and Igor Surovtsev ${ }^{1}$ \\ ${ }^{1}$ Voronezh state technical University, Moskovsky Prospekt 14, Voronezh, 394026, Russia
}

\begin{abstract}
In this paper, we propose a Supplement to the theory of the emergence of innovations in long Kondratiev cycles. The regularities of the emergence of crisis phenomena and the contribution of J. Schumpeter to the development of the innovative economy are considered. A comparative analysis of Marketing strategies is carried out. Schumpeter and N. D. Kondratiev, identified distinctive factors and common combinations. The stages of long wave cycles over several decades are analyzed. The analysis revealed innovative peaks that occur in the middle of the depression phase, followed by stages of economic activity growth after a certain period of time. The authors studied the theory of the trigger effect of depression, similar to the trigger action, which results in an avalanche of innovation growth. To confirm the theory of the existence of the "trigger effect of depression", statistical data on the number of patents and applications filed in Russia and the United States for several decades, which are indicators of innovation activity, are presented. The authors put forward a hypothesis about the possibility of describing the periodic change in the number of innovations over time using "parametric resonance" models and the Mathieu equation.
\end{abstract}

\section{Introduction}

At the beginning of the XX century, the term "innovation" was first introduced into economic theory and scientific circulation as a new economic category that defines changes in order to introduce, produce, and use new products, markets, and forms of company organization. J. Schumpeter was the first to reveal this concept and theoretically justified the importance of innovations for market competition based on advances in science and technology. By definition, Y. Schumpeter, innovation is a new combination of production factors, motivated by an entrepreneurial spirit [1].

It is known that N. D. Kondratiev made a great contribution to the development of innovative strategies, justifying innovative development as the idea of commercial and industrial cycles, which subsequently led to the development of the theory of large cycles, presented in Fig.1. N. D. Kondratev showed that innovations are distributed unevenly over time, have a wave-like character and consist of four phases that are synchronously repeated

\footnotetext{
* Corresponding author: sof1355@yandex.ru,
} 
after each cycle. Before the beginning of the rising wave of each cycle, there are profound changes in technology, which are preceded by scientific discoveries and inventions [2].

Presenting the doctrine of large cycles of conjuncture of approximately half a century duration, Kondratev justified the natural connection of the "up" and" down " waves of these cycles with the waves of technical inventions and their practical use [3]. "Before the beginning of the upward wave of each large cycle, and sometimes at its very beginning, there are significant changes in the basic conditions of economic life of society. These changes are usually expressed (in one or another combination) in profound changes in production techniques and volume (which in turn are preceded by significant technical inventions and discoveries), in changes in the conditions of monetary circulation, in the strengthening of the role of new countries in world economic life". In fact, the quote refers to a wave of technological and economic innovations [4].

Kondratev links these waves to radical changes in other areas of society. Periods of rising waves of large cycles, as a rule, he notes, are much richer in major social upheavals and upheavals in the life of society (revolutions, wars) than periods of downward waves. Thus, he continues, both wars and social upheavals are included in the rhythmic process of development of large cycles and are not the initial forces of this development, but the form of its manifestation. But once they arise, of course, they in turn have a powerful and sometimes perturbating effect on the pace and direction of economic dynamics [5].

Each long wave consists of four phases: rise, fall, crisis, and recovery. Each subsequent phase occurs as a result of cumulative processes accumulated in the previous phase.

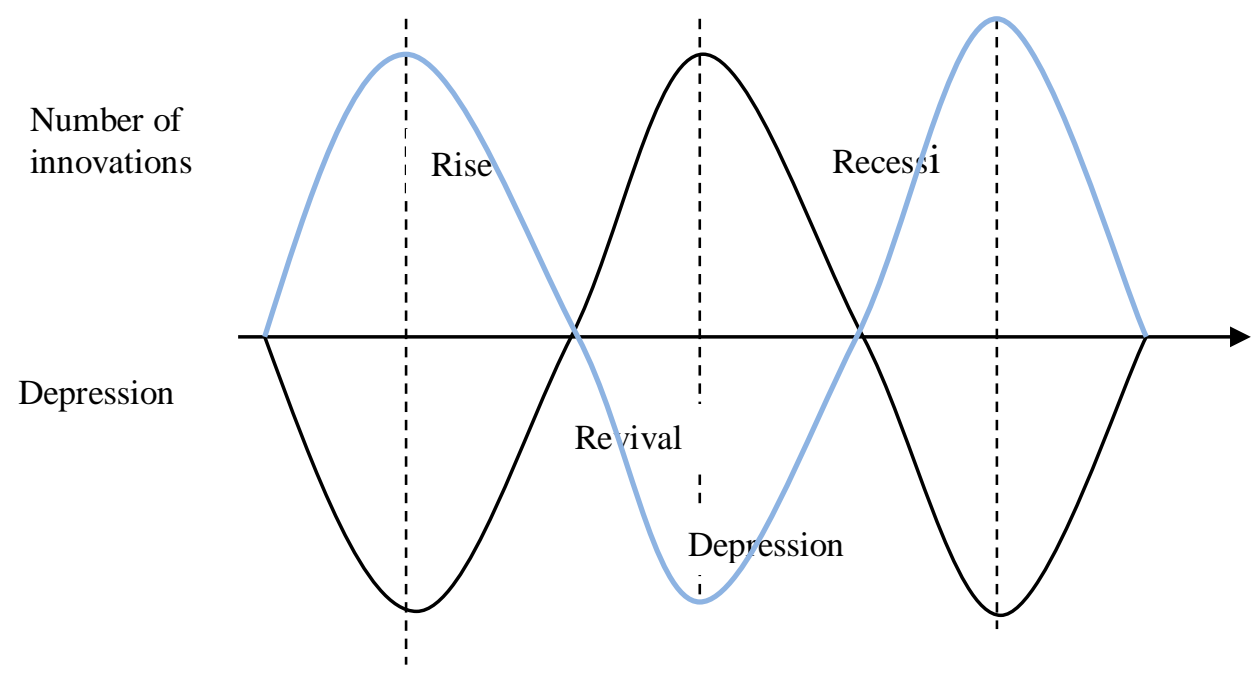

Fig.1. Four-phase of the Kondratiev cycle.

\section{Materials and methods}

Kondratiev's huge contribution to the development of this problem was highly appreciated by Experts. Schumpeter and other Western scientists, and since the late 1930s, long cycles have been called "Kondratiev cycles". Thus, we can conclude that N. D. Kondratiev laid the foundations of a General theory of innovation, covering not only technical development and 
the economy, but also the socio-political sphere, as well as revealing the mechanism of interaction of innovations in various spheres of society [6].

However, Schumpeter's contribution to economic theory is precisely that he explores the factors that "blow up" the equilibrium of the market system from within. These internal factors are new production combinations that determine the dynamic changes in the economy. Schumpeter identifies several types of fundamentally new combinations of factors of production (Fig.2). The authors studied the innovative theories of J. Schumpeter and N. D. Kondratiev, distinctive factors and General combinations.

New combinations of factors of production are called "innovations". It should be emphasized that in Schumpeter's terminology, "innovation" is not synonymous with the word "invention" since business activity is associated with the use of existing tools, and not with the creation of new ones. The possibilities of new use of funds are in excess of themselves, they can be known. But, as Schumpeter suggests, these are" dead " possibilities. The entrepreneur, on the other hand, implements them in reality, overcoming technological and financial difficulties and thus opening up new ways of making a profit, which should be considered as an excess over the income that has been established in the course of the cycle. And it is such an entrepreneur, whose function is to implement a new combination of factors of production, that is assigned a particularly important role in Schumpeter's concept of economic development [7]. 


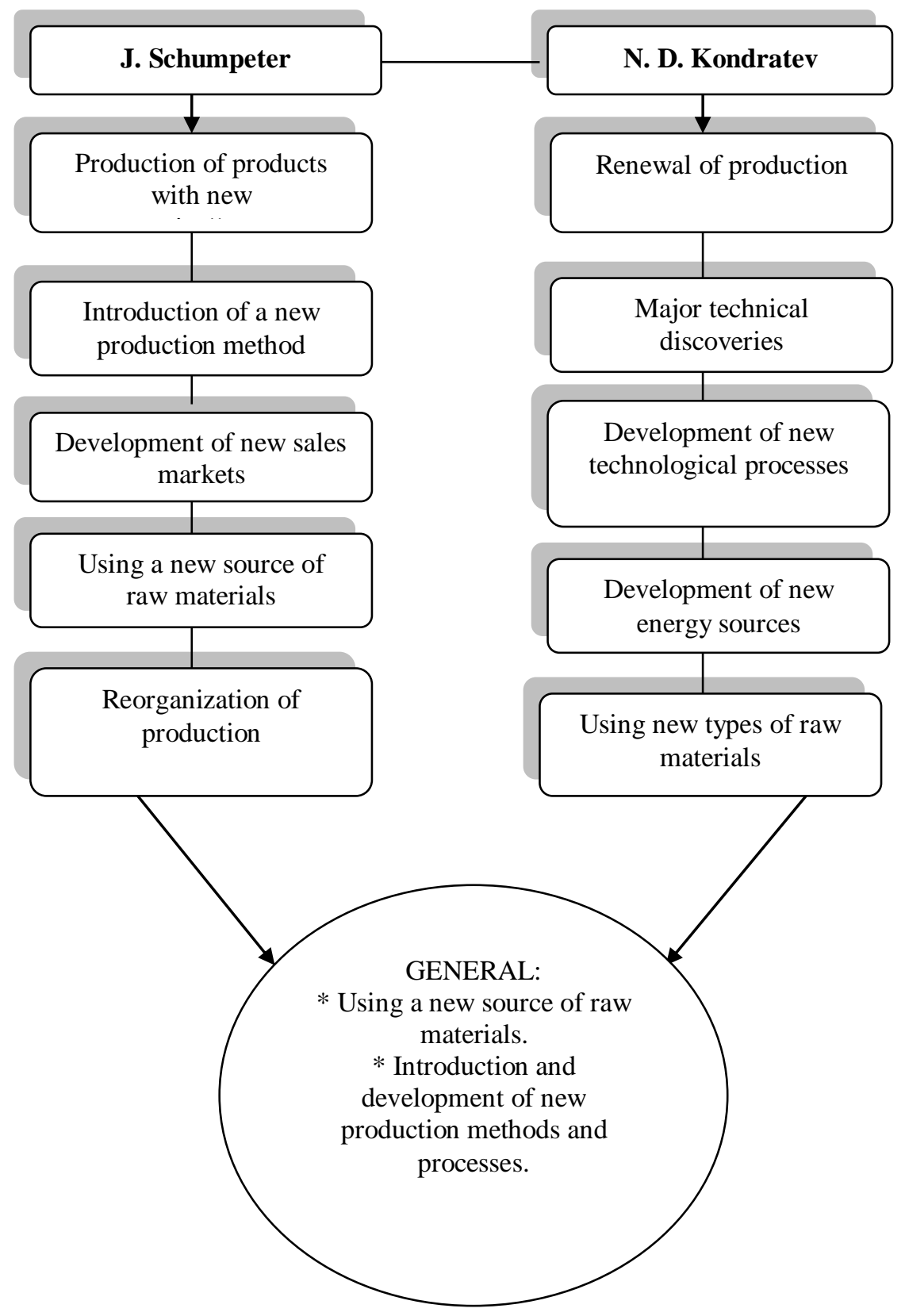

Fig.2. Comparative analysis of Marketing strategies. Schumpeter and N. D. Kondratiev.

It should be emphasized that entrepreneurship, according to Schumpeter, is a special gift, a property of human character that is absolutely independent of class and social affiliation. This type of character is distinguished by the following features:

- self-reliance;

- risk preference;

- the value of your own independence; 
- focus on your own opinion;

- the need to achieve success, despite the fact that the self-value of money for him is small;

- and as a key quality of the entrepreneur — the desire for innovation [8].

\section{Results}

It is revealed that during periods of depression, the market of the economy is most prone to the emergence of innovations, since depression makes us look for ways to survive, and innovations and their active application can provide such ways [9, 10]. This unusual phenomenon is called the trigger effect of depression. Interestingly, it is depression that triggers the innovation process $[11,12]$.

Examples of depression in different years and their analysis are shown in fig. 3.

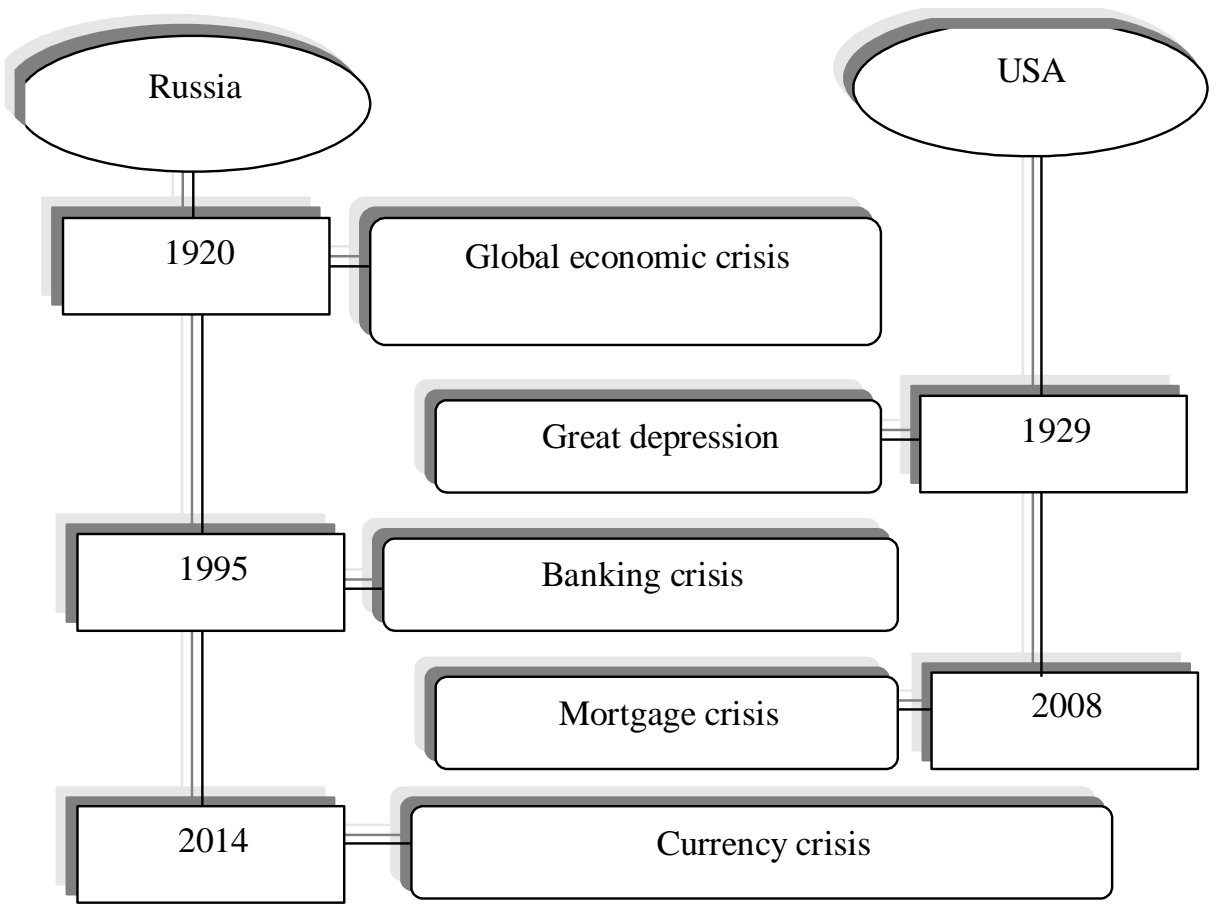

Fig.3. Moments of depression in Russia and the United States.

The analysis revealed innovative peaks that occur in the middle of the depression phase, followed by stages of economic activity growth after a certain period of time [13].

The analysis of patent activity in Russia and the United States is performed to confirm the theory of the "trigger effect of depression", where the number of registered patents and patent applications is given as indicators of patent activity [14]. Statistical data on the number of innovations in the period before crises, during crises, and post-crisis years are presented in the table. 1. 
Table. 1. Number of patents in Russia and the United States.

\begin{tabular}{|c|c|c|c|c|c|c|c|c|c|}
\hline $\begin{array}{c}\text { Number } \\
\text { of patents: }\end{array}$ & 1919 & 1920 & 1921 & 1994 & 1995 & 1996 & 2013 & 2014 & 2015 \\
\hline Russia & 22036 & 24072 & 21152 & 21458 & 26795 & 24596 & 21056 & 23065 & 21489 \\
\hline $\begin{array}{c}\text { Number } \\
\text { of patents: }\end{array}$ & 1928 & 1929 & 1930 & 2007 & 2008 & 2009 & - & - & - \\
\hline USA & 15485 & 16248 & 13269 & 8952 & 13677 & 7426 & - & - & - \\
\hline
\end{tabular}

Statistics on the number of patents from different years in Russia and the United States and the peaks of innovation activity during the crisis years are shown in fig. 4 .

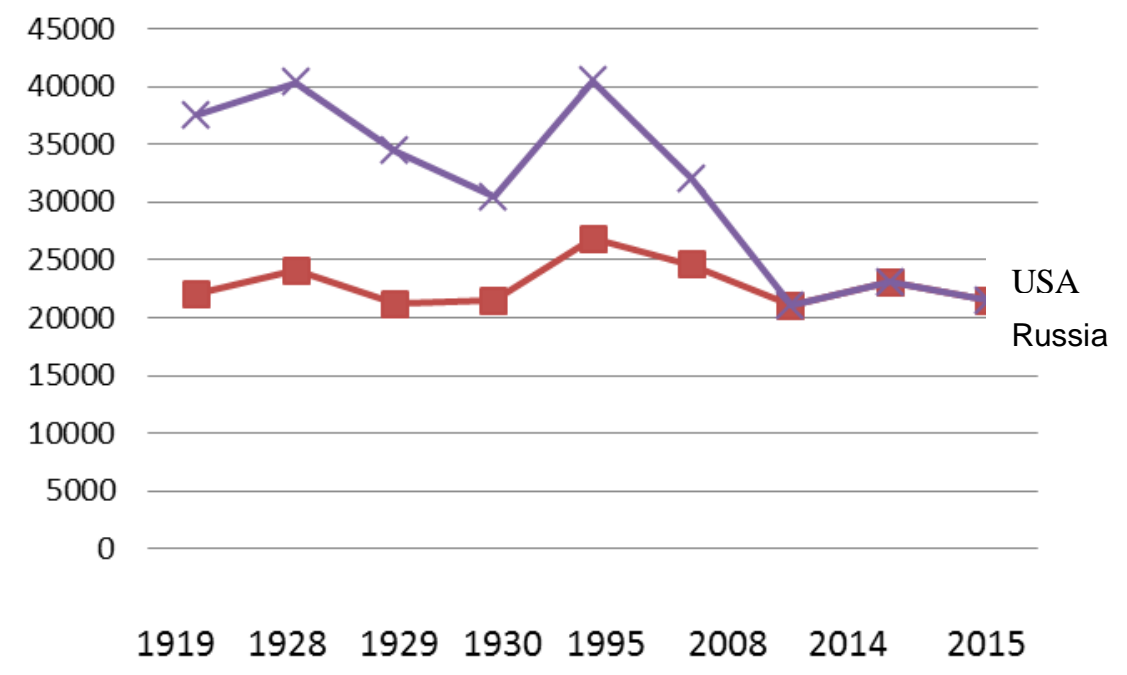

Fig.4. Peaks of innovation activity in crisis years. 
Trigger (from the English. trigger trigger) in a General sense means "trigger"). The trigger effect is a rapid transition of the system to another state under the influence of internal avalanche-like processes. This conversion begins when the trigger system is affected by a signal that is greater than a certain minimum level (threshold). The trigger effect is based on the trigger of a gun, a trap, and a wick in a barrel of gunpowder. For a trigger effect, the following phenomenon is typical: the shape of the trigger device does not depend on the strength and size of the internal crisis and the factor causing it, but on the magnitude of the impact on it, so long as it exceeds the "trigger threshold". The minimum of a sufficient amount of the exploding factor depends on the degree of tension of relations within the system [15]. The closer it is to the possible threshold, the lower the value of the blasting factor, so in cases where the degree of tension is low, the nature and strength of the signal can have a big impact.

As a hypothesis put forward a hypothesis about the resonance nature of the triggering of such trigger.

It is known that the classical resonance is associated with a sharp increase in the amplitude of stationary oscillations, when the frequency $\omega$ coincides with certain values of $\omega_{-} i$ characteristic of this system.

Since we are talking about cyclical processes, alternating crises, revivals, etc., it is advisable to consider a more complex resonance - parametric-as the reason for the avalanche effect of increasing the number of innovations.

When describing the avalanche-like growth of innovations, we are talking about rather complex cyclical processes, extended over time and associated with the effects of accumulation.

The processes of avalanche growth of innovations are not even related to specific values, but to entire areas (intervals) of parameter values that affect the described systems.

Therefore, in the authors ' opinion, the analogy with the so-called "parametric resonance"is more suitable for describing the avalanche-like growth of innovations.

The parametric resonance is closely related to the well-known Mathieu equation, for which the regions of stability and instability of the solution are known, illustrated by the Ains-Strett diagrams (Fig. 5).

Getting into the area of instability just means the sharp (and often unlimited) increase in the solution, for example, the amplitude of fluctuations in a process, which we propose to associate with the avalanche growth of innovations.

"Parametric resonance" is related to the solution of the equation of the form:

$$
\frac{d^{2} x}{d t^{2}}+\omega^{2}(t) x=0 .
$$

Here, the variable $x$ can make sense of the amplitude of some oscillatory process. We can consider $\mathrm{x}$ as the value of the number of innovations that changes periodically over time.

In our case, we can consider the equation $\omega(t)$, which is usually considered as a frequency in physical interpretations, as a time-varying (and, accordingly, time-dependent $\mathrm{t}$ parameter), which itself is a complex combination of many other parameters that determine the development of innovations, i.e., the frequency of the process. $\omega(t)=F\left(P_{1}, P_{2, \ldots}, P_{n}\right)=F\left(P_{1}(t), P_{2}(t), \ldots P_{n}(t)\right)$

In the special case of periodic dependence $\omega(t)$, equation (1) is a well-known hill equation, and in the case of a harmonic dependence $\omega(\mathrm{t})$ - a special case of the Mathieu equation, which in canonical form has the form: 


$$
\frac{d^{2} x}{d t^{2}}+[a-2 q \cos (2 x)] y=0,
$$

where а и $\mathrm{q}$ - the parameters on which the behavior of the solution depends (stable or unstable), falling into the region of instability, just means the sharp increase in the amplitude of oscillations, which we associate with an avalanche of innovations in the process of Kondratiev cycles.

This dependence is illustrated by the well-known Ains-Strett diagram, one of the variants of which is shown in fig.5. Here $\gamma$ - parameter similar to the parameterq in equation (2), $\lambda$ - parameter similar to parameter $\mathrm{a}$ in the equation (2).

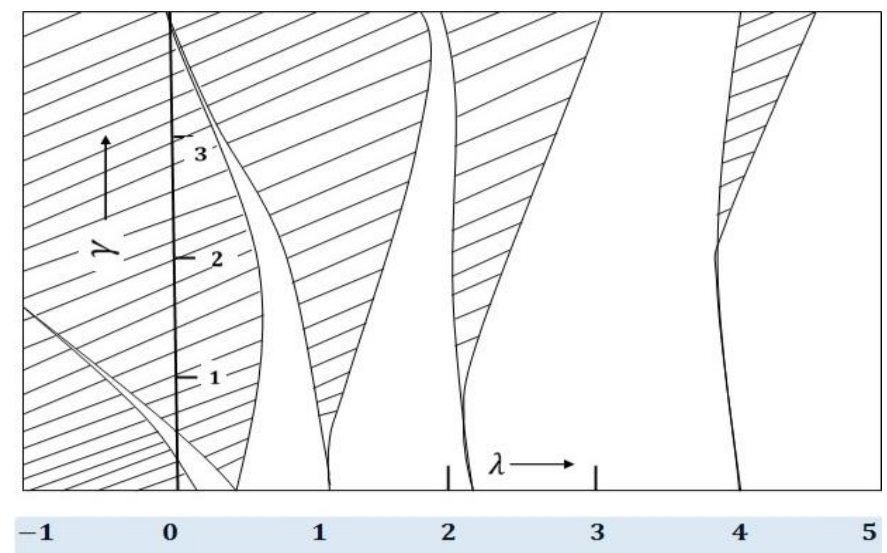

Fig.5. Stability diagram for the Mathieu differential equation. Areas of instability are highlighted with hatching.

The shaded areas correspond to the resonance (instability and strong growth of the solution, corresponding to the process of avalanche growth of innovations). Defining a specific type of dependency $\omega(t)$ as a combination of parameters, $P_{1}(t), P_{2}(t), \ldots, P_{n}(t)$, defining the development of innovations, and clarifying the number of these parameters and their socio-economic meaning is a very promising task and will be developed in the future works of the authors.

If we go back to the equation (1) $\frac{d^{2} x}{d t^{2}}+\omega^{2}(t)=0$ and let's consider one of the possible special cases when $\omega^{2}(t)=\omega_{0}^{2}\left[1+h \cos \left(\omega_{0}+\varepsilon\right) t\right]$, then equation (1) will take the form:

$$
\frac{d^{2} x}{d t^{2}}+\omega_{0}^{2}\left[1+h \cos \left(\omega_{0}+\varepsilon\right) t\right) x=0
$$

where $\omega_{0}$ - the frequency of harmonic oscillations, the amplitude of the harmonic variations of the frequency $\mathrm{h} \ll 1$, constant $\varepsilon \ll \omega_{0}$ - small frequency variation. By properly changing the start of the countdown $h$ you can choose positive, so without limiting generality, we assume that $h>0$.

\section{Discussions}

Let's ask the question: at what values of the parameter $\varepsilon$, the oscillation amplitude increases dramatically, i.e. the solution $\mathrm{x}(\mathrm{t})$ increases indefinitely? 
Recall that we interpreted $x(t)$ above as the number of innovations that changes over time. Note that in contrast to the ideal mathematical model of unlimited growth $x(t)$, in reality, the increase in the number of innovations will not be unlimited, but there will be a sharp avalanche, but still a limited increase in the number of innovations.

It is easy to show how this is done, for example in [12] what it will be in the case of: $\frac{-5}{24}<\frac{\varepsilon}{h^{2} \omega_{0}}<\frac{1}{24}$, that is, in the case considered $\omega(t)=\omega_{0}^{2}\left[1+h \cos \left(\omega_{0}+\varepsilon\right) t\right]$ in contact with the relations of parameters $\frac{\varepsilon}{h^{2} \omega_{0}}$ in the range $\left(-\frac{5}{24} ; \frac{1}{24}\right)$ there will be an unlimited growth of solution $x(t)$, which can be interpreted as a sharp increase in the number of innovations.

As already mentioned above, the definition of a specific number and clarification of the socio-economic meaning and role of the parameters included in equations (1-3) is very promising and will be further developed in the authors ' works.

\section{Conclusion}

In recent times by various scholars there is a growing interest in the theory of long waves of Kondratiev.

The authors give comparative features, differences, and General combinations in the theory of development of Kondratiev and J. Schumpeter. The regularities of the emergence of crisis phenomena and the contribution of J. Schumpeter to the development of the innovative economy are considered.

In this paper, we analyzed the stages of long wave cycles over several decades, which allowed us to systematize the crisis phenomena and identify the peaks of innovation activity. It is proved that the active emergence of innovations, called by us "avalanche growth of innovations", falls in the middle of the depression phase. This leads to stages of significant growth in economic activity at certain intervals.

The authors justified and expanded the concept of "trigger effect of depression", which can also be referred to as a" trigger", i.e. certain actions leading to the transition of the system to a new state. To confirm the theory of the existence of the "trigger effect of depression", statistical data on the number of patents and applications filed in Russia and the United States for several decades, which are indicators of innovation activity, are presented.

The authors put forward a hypothesis about the possibility of describing the periodic change in the number of innovations over time using "parametric resonance" models and the Mathieu equation.

\section{References}

1. N. S. Tülüce, A. K. Yurtku, Procedia - Social and Behavioral, 720-728 (2015) doi: 10.1016/j.sbspro.2015.10.146

2. J. Kurth, doi: 10.1016/j.orbis.2011.04.017

3. B.-A. Schuelke-Leech, Technological Forecasting and Social Change, 261-274 (2018) doi:10.1016/j.techfore.2017.09.033

4. P.-A. Jouvet, C. de Perthuis, International Economics, 134, 29-55 (2013) doi: 10.1016/j.inteco.2013.05.003

5. J. Köhler, Environmental Innovation and Societal Transitions, 3, 1-15 (2012) doi: 10.1016/j.eist.2012.04.001 
6. L. Costa Ribeiro, L. Gomez de Deus , P. Mendes Loreiro , E. da Motta, et al., Structural Change and Economic Dynamics, 43, 51-61 (2017) doi: 10.1016/j.strueco.2017.07.001

7. K. Wersching, Journal of Economic Behavior \& Organization, 75, 482-493 (2010) doi: 10.1016/j.jebo.2010.05.005

8. L. S. M. Miranda, C. Lima, Technological Forecasting and Social Change, 78, 14451470 (2011) doi: 10.1016/j.techfore.2011.04.001

9. M. Coccia, Technological Forecasting and Social Change, 77, 730-738 (2010) doi: 10.1016/j.techfore.2010.02.003

10. D. Loorbach, J. Wittmayer, et al., Environmental Innovation and Societal TransitionsIn press (2020) doi: 10.1016/j.eist.2020.01.009

11. J. Juliao-Rossi, C. Forero-Pineda, et al., Journal of Business ResearchIn press (2019) doi: 10.1016/j.jbusres.2019.11.026

12. M. C.W. Solheim, R. Boschma, S. J. Herstad, Research policy, 49, 103856 (2020) doi: 10.1016/j.respol.2019.103856

13. D. B. Audretsch, M. Belitski, European Economic Review, 123, 103391 (2020) doi: 10.1016/j.euroecorev.2020.103391

14. S. S. Gulshan, doi: 10.1016/j.sbspro.2011.10.527 